\title{
A gauge invariant chiral unitary framework for kaon photo- and electroproduction on the proton
}

\author{
Peter C. Bruns ${ }^{* \dagger}$ \\ Helmholtz-Institut für Strahlen-und Kernphysik and Bethe Center for Theoretical Physics \\ Universität Bonn \\ Nußalle 14-16, 53115 Bonn (Germany) \\ E-mail: bruns@hiskp.uni-bonn.de
}

We present a gauge-invariant approach to photoproduction of mesons on nucleons within a chiral unitary framework

6th International Workshop on Chiral Dynamics

July 6-10 2009

Bern, Switzerland

\footnotetext{
* Speaker.

$\dagger$ I would like to thank the organizers for the invitation to the conference. Moreover, I want to thank Bugra Borasoy, Ulf-G. Meißner and Robin Nißler for a fruitful collaboration on the subject. This research is part of the EU Integrated Infrastructure Initiative HadronPhysics2 Project. Work supported in part by DFG (SFB/TR 16, "Subnuclear Structure of Matter") and by the Helmholtz Association through funds provided to the virtual institute "Spin and strong QCD" (VH-VI-231).
} 


\section{Introduction}

A successful theoretical approach to meson-baryon dynamics is provided by chiral unitary methods, see e.g. [1-5]. In this framework the chiral effective Lagrangian is utilized to derive, for example, the interaction kernel in a Bethe-Salpeter equation (BSE) which iterates meson-baryon rescattering to infinite order. The BSE generates resonances dynamically, hence, without their explicit inclusion the importance of resonances can be studied. Chiral unitary approaches have been implemented quite successfully for photoproduction processes, see e.g. refs. [6-11], but as a simplification only those diagrams were taken into account where the photon is absorbed first and then the produced meson-baryon pair undergoes final state interaction. This simplified treatment violates, in general, gauge invariance.

The main goal of the present contribution is the construction of a minimal approach to meson photoproduction based on the chiral effective Lagrangian which is exactly unitary and gauge invariant. The presented method fulfills these important requirements from field theory, while at the same time any subset of diagrams cannot be omitted as this would violate unitarity or gauge invariance. In this first study, we restrict ourselves to the chiral effective Lagrangian at leading order.

This article is organized as follows. In the next section, the effective Lagrangian and the BetheSalpeter formalism are introduced. The gauge invariant extension to photo- and electroproduction processes is discussed in sect. 3. Section 4 contains the comparison with experimental data on kaon photoproduction. We summarize our findings in sec. 5, where we will also give a brief outlook on forthcoming work on this subject.

\section{Bethe-Salpeter equation}

The chiral effective Lagrangian incorporates symmetries and symmetry-breaking patterns of QCD in a model-independent way, in particular chiral symmetry and its explicit breaking through the finite quark masses. By expanding Green functions in powers of Goldstone boson masses and small momenta a chiral counting scheme can be established. However, the strict perturbative chiral expansion is only applicable at low energies, and it certainly fails in the vicinity of resonances. In this respect, the combination of the chiral effective Lagrangian with non-perturbative schemes based on coupled channels and the Bethe-Salpeter equation (BSE) have proven useful both in the purely mesonic and in the meson-baryon sector [1-5]. Such approaches extend the range of applicability of the chiral effective Lagrangian by implementing exact two-body unitarity in a nonperturbative fashion and generating resonances dynamically.

Here we restrict ourselves to the meson-baryon Lagrangian at leading order,

$$
\mathscr{L}_{\phi B}^{(1)}=\left\langle\bar{B}\left([i \not D, B]-m_{0} B\right)\right\rangle-\frac{D}{2}\left\langle\bar{B} \gamma^{\mu} \gamma_{5}\left\{u_{\mu}, B\right\}\right\rangle-\frac{F}{2}\left\langle\bar{B} \gamma^{\mu} \gamma_{5}\left[u_{\mu}, B\right]\right\rangle,
$$

where the matrix $B$ collects the ground state baryon octet. All the definitions employed here are rather standard and can be found in [12]. In our numerical work, we use the coupling constants $D=0.8, F=0.46[13]$ in the leading order meson-baryon Lagrangian. The meson decay constants occuring in $u_{\mu}$ will undergo a special treatment mentioned below.

By expanding the chiral connection in powers of the meson fields, one derives from the effective Lagrangian the leading order $\phi^{2} \bar{B} B$ vertex (the so-called 'Weinberg-Tomozawa' (WT) term), which 


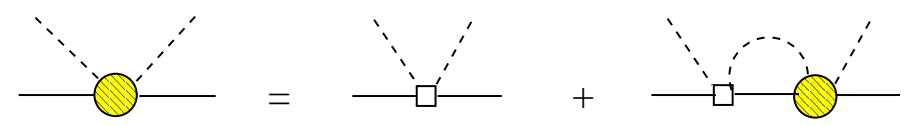

Figure 1: Graphical illustration of the BSE for meson-baryon scattering. The filled circle represents the full scattering matrix and the open square the driving meson-baryon vertex.

we use as the driving term in our Bethe-Salpeter equation ( $\phi$ denotes the ground state octet of meson fields). One finds for the corresponding potential $V$ (which is the WT-vertex graph multiplied by $i$ )

$$
V^{b j, a i}\left(q_{2}, q_{1}\right)=g^{b j, a i}\left(q_{1}+q_{2}\right) \text {. }
$$

Here, $q_{1}$ and $q_{2}$ are the four-momenta of the incoming and the outgoing meson, respectively, and the coupling constants are summarized as a matrix in channel space, with the entries

$$
g^{b j, a i}=-\frac{1}{4 F_{j} F_{i}}\left\langle\lambda^{b^{\dagger}}\left[\left[\lambda^{j \dagger}, \lambda^{i}\right], \lambda^{a}\right]\right\rangle,
$$

where $\lambda^{a}$ are the generators of the $S U(3)$ Lie-Algebra in the physical (particle) basis. In this representation, a double index $b j$ specifies a particular channel consisting of a baryon $b$ and a meson $j$. In the above expressions, $a i$ specifies the channel of the incoming particles, while $b j$ labels the outgoing meson-baryon state. Note that we use different values for the meson decay constants $F_{i}$ (where again $i$ labels the meson in the corresponding channel), instead of the meson decay constant in the chiral limit. In practice, these three constants $F_{\pi}, F_{K}, F_{\eta}$ will be used as fit parameters, which are allowed to vary in a reasonable range, to be specified in sec. 4 . We refer to the latter section for a discussion of this issue.

The baryon and the meson propagator, $i S$ and $i \Delta$, are summarized as (diagonal) matrices in channel space. We can now write down the integral equation for the meson-baryon scattering amplitude $T^{b j, a i}$ in a rather compact form (suppressing the channel indices, but remembering the matrix character of the various amplitudes):

$$
T\left(q_{2}, q_{1} ; p\right)=V\left(q_{2}, q_{1}\right)+\int \frac{d^{d} l}{(2 \pi)^{d}} V\left(q_{2}, l\right) i S(\not p-l) \Delta(l) T\left(l, q_{1} ; p\right) .
$$

Here, $p \equiv p_{1}+q_{1}=p_{2}+q_{2}$ is the overall momentum, where $p_{1}$ and $p_{2}$ are the four momenta of the incoming and outgoing baryon, respectively. The BSE is illustrated in fig. 1. Note that we use dimensional regularization throughout. The solution of the BSE is written out in detail in [12]. Even more details on the properties of the BSE and its solution can be found in [14]. We note in passing that we do not set the external momenta occuring in the potential $V$ on the mass shell when we iterate it in the BSE. In contrast, we evaluate all Feynman graphs in the way dictated by the rules of field theory. This will prove very convenient when we come to the implementation of gauge invariance in the full (photoproduction) amplitude, which then becomes a rather straightforward procedure as outlined below. The (off-shell) solution of the BSE has the form

$$
\begin{aligned}
T\left(q_{2}, q_{1} ; p\right)= & q_{2} \not p q_{1} T_{1}(p)+q_{2} q_{1} T_{2}(p)+\not p q_{1} T_{3}(p)+q_{2} \not p T_{4}(p)+q_{1} T_{5}(p) \\
& +q_{2} T_{6}(p)+\not p T_{7}(p)+T_{8}(p) .
\end{aligned}
$$


The scalar coefficient functions $T_{n}(p)$ are matrices in channel space, and depend only on the variable $p^{2}$. They will enter the calculation of the various photo- and electroproduction amplitudes in the next section. To obtain the complete on-shell meson-baryon scattering amplitude, this expression has to be sandwiched between baryon spinors $\bar{u}\left(p_{2}\right) \ldots u\left(p_{1}\right)$. Two-body unitarity in the space of meson-baryon channels is guaranteed by the fact that $T$ is the solution of the BSE with hermitian potential (interaction kernel) $V$. Our approach to photoproduction presented in the next section will be such that unitarity is also satisfied when the photon is coupled to the meson-baryon bubble chain summed up by means of the BSE, using the method outlined in [15], and described in the next section.

\section{Photo- and electroproduction}

The Bethe-Salpeter approach discussed in the last section (or the Lippmann-Schwinger equation in the non-relativistic framework) can be implemented in electroproduction processes of mesons on the nucleon. In previous work, the electromagnetic meson production on the nucleon was calculated at tree level and the produced meson-baryon pair was subject to final-state interactions $[1,8]$. As the photon does not couple to all intermediate states of the meson-baryon bubble chain, gauge invariance is in general violated and must be restored via artificial manipulations. Therefore it seems desirable to develop a formalism which implements the principles of gauge invariance and unitarity in a most natural and straightforward manner. We follow here the path which we have already outlined in [15] in rather general terms, for the case of a photon coupling to a meson-baryon scattering amplitude. In this work, we shall be more explicit in evaluating the contributions to the various amplitudes in question. Our approach for constructing a unitary and gauge invariant electroproduction amplitude decomposes into two major steps:

(1) Fix the hadronic part of the amplitude by making use of a BSE to implement exact two-body unitarity.

(2) Couple the photon to the 'hadronic skeleton' constructed in step (1) wherever possible, i.e. to all external and internal lines describing the propagation of the involved particles as well as to (momentum-dependent) vertices.

The procedure of step (2), which is the most natural way to guarantee gauge invariance of the amplitude, leads to contributions that were usually not considered in chiral unitary approaches involving electromagnetic interactions. The importance of these additional contributions which render the electroproduction amplitude gauge invariant can also be quantified within the scheme utilized here.

The leading order (tree level) $\bar{B} \phi B$ amplitude is easily derived from the leading order Lagrangian of eq. (2.1). Employing this vertex insertion, and the meson-baryon scattering amplitude derived from the BSE, we can construct the 'hadronic skeleton' mentioned above, and illustrated in fig. (2). In order to complete step (1), we still have to specify which meson-baryon channels contribute in the framework of our electroproduction model. In this first study, we choose to consider only the ground-state octets of mesons and baryons, respectively. Moreover, from the topology of the 


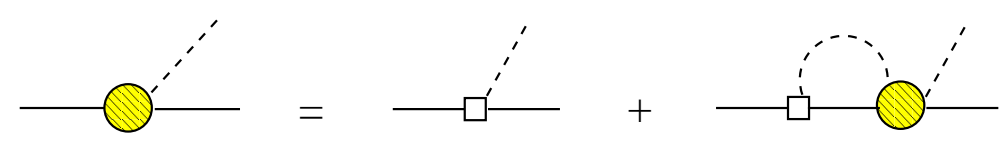

Figure 2: The dressed meson-baryon vertex in our model amplitude. The filled circle (open square) denotes the full (tree level) meson-baryon interaction.

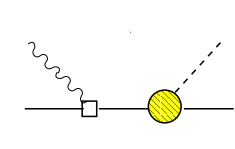

A

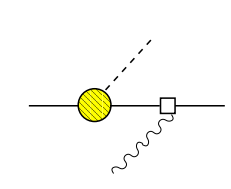

B

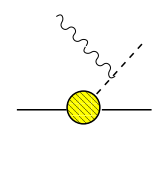

$\mathrm{C}$

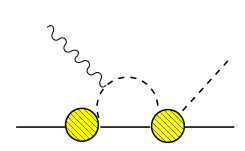

$\mathrm{D}$

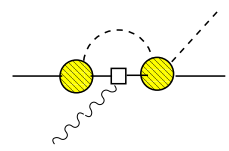

$\mathrm{E}$

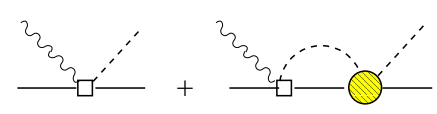

$\mathrm{F}$

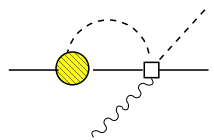

G

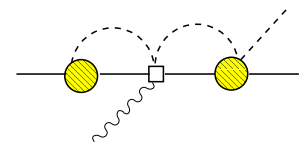

$\mathrm{H}$

Figure 3: Classes of diagrams for kaon production off the nucleon in the approximation utilized here.

hadronic part of the Feynman graph in fig. 2 we can conclude that the meson-baryon pairs must have the charge and strangeness quantum numbers of the proton. This limits the number of channels to six:

$$
p \pi^{0}, n \pi^{+}, p \eta, \Lambda K^{+}, \Sigma^{0} K^{+}, \Sigma^{+} K^{0}
$$

The limitation to these channels can only occur because our amplitude is not crossing-symmetric, otherwise more channels with different quantum numbers must be considered. The violation of crossing-symmetry is a drawback of the BSE method which we use to iterate the rescattering graphs.

By now, we have finished the first part (step (1)) of our program. Our next task is to couple the photon to the hadronic part of the amplitude in a gauge-invariant fashion. Inserting the photon coupling at every possible place leads to the set of diagrams displayed in fig. 3. Again, explicit expressions for all those Feynman graphs are given in [12].

Having finished the construction of the electroproduction amplitude, we return to the issue of unitarity. The crucial observation here is that every electroproduction amplitude $\mathscr{M}^{\mu}(q, k ; p)$ which may be written as (see also fig. 4)

$$
\mathscr{M}^{\mu}(q, k ; p)=\mathscr{M}_{0}^{\mu}(q, k ; p)+\int \frac{d^{d} l}{(2 \pi)^{d}} \mathscr{T}(q, l ; p) i S(\not p-\not) \Delta(l) \mathscr{M}_{0}^{\mu}(l, k ; p)
$$




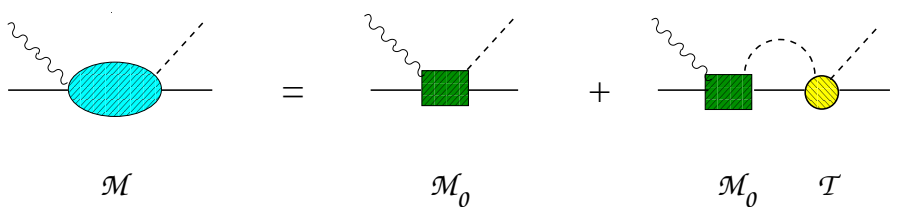

Figure 4: Illustration of the integral equation (3.2) satisfied by our electroproduction amplitude.

obeys the requirement of two-body unitarity in the subspace of meson-baryon channels. Here, $\mathscr{T}$ is an amplitude for meson-baryon scattering that solves a BSE of the type of eq. (2.4) and $\mathscr{M}_{0}^{\mu}$ is a real kernel. The proof proceeds in close analogy to the one presented in sec. 5 of [15]. It is straightforward to convince oneself that our model amplitude is of the form described by the foregoing equation. We also have to show that the sum $\mathscr{M}^{\mu}$ of the specified graphs is gauge invariant by proving $k \cdot \mathscr{M}=0$ for on-shell mesons and baryons. Although this might be obvious from our construction, we have included an explicit proof of this statement in [12].

\section{Results}

Using our model amplitude, we have performed an overall $\chi^{2}$ fit to available photoproduction and pion-induced data on the proton near the respective thresholds. In more detail, we fit the differential cross sections for photoproduction on the proton into the $K^{+} \Lambda, K^{+} \Sigma^{0}, K^{0} \Sigma^{+}$final states as well as of $\pi^{-} p \rightarrow K^{0} \Lambda, K^{0} \Sigma^{0}$. Inspection of the differential cross sections reveals that already at moderate energies away from threshold $p$ - and $d$-waves become increasingly important. Since our approach, which is based on the Weinberg-Tomozawa interaction kernel, generates mainly $s$ waves (with the exception of the t-channel exchange graph $\mathrm{C}$ in fig. 3) and thus does not provide a realistic description for higher partial waves, we expect it to be valid only in the near-threshold regions. We have thus restricted our fits to energy values for which the differential cross sections are dominated by $s$-waves, i.e. center-of-mass energies of about $1.80 \mathrm{GeV}$ corresponding to photon lab momenta of about $1.25 \mathrm{GeV}$ or pion lab momenta of about $1.23 \mathrm{GeV}$. Still, we will be able to extract interesting information from such investigations, in particular, we can study in detail the commonly appearing approximations made in the literature as already mentioned in the introduction. The free parameters in our approach are, on the one hand, the three meson decay constants $F_{\pi}, F_{K}, F_{\eta}$ which we vary separately within realistic bounds, as the $\mathrm{SU}(3)$ symmetry-breaking differences between them are beyond our working precision of the effective potential. More precisely, SU(3) symmetry breaking is generated by various higher order terms in the meson-baryon (meson) Lagrangian starting at chiral order two (four). Since these contributions are not included in the leading order WT kernel, we simulate such effects by allowing variations in the various meson decays constants. This, of course, will no longer be done when the higher order terms in the interaction kernel have been included. One observes that the fitted decay constants tend to larger values reducing the strength of the Weinberg-Tomozawa interaction. This is consistent with findings in earlier chiral unitary studies, i.e. the WT interaction in many cases produces too strong $s$-waves, see e.g. [8]. We allow $F_{\pi}, F_{K}$, and $F_{\eta}$ to vary between $70 \mathrm{MeV}$ and $150 \mathrm{MeV}$. On the other hand, we fit the four different isospin-symmetric scales $\mu$ in the loop integrals. For the best fits their values 
are roughly in accordance with the natural size estimate of ref. [2] (although most of them turn out to be somewhat large).

We have included in our fit data on differential cross sections of the photoproduction processes $\gamma p \rightarrow K^{+} \Lambda, K^{+} \Sigma^{0}, K^{0} \Sigma^{+}$. Some results are displayed in figs. 5 and 6 , respectively. A few remarks are in order: The SAPHIR and CLAS data on charged kaon photoproduction show some inconsistencies at forward angles, but this can not be resolved within the approximations made here. Also, the very different shape of the differential cross sections for the $K^{+} \Lambda$ and $K^{+} \Sigma^{0}$ final states can be traced back to the isospin selectivity of the $\Lambda$, see also ref. [16].

To summarize, the results presented here show a reasonable agreement with data on photonand pion-induced reactions close to threshold, but more realistic interaction kernels and higher partial waves are required to obtain better agreement with data, in particular away from threshold. This is however beyond the scope of the present investigation. We have also examined gauge invariance violations encountered in previous chiral unitary approaches which only took a subset of the diagrams in fig. 3 into account. One clearly observes that violations of gauge invariance are sizable, although this effect could, in principle, be concealed numerically by readjusting the parameters of the approach. This indicates that the additional contributions which render the photoproduction amplitudes gauge invariant and were omitted in previous work are not negligible and must be taken into account.

In order to be able to compare our results with previous chiral unitary approaches we have also worked in the approximations employed in these investigations, see e.g. [8]. One notes that the effects of these approximations can indeed be sizable. Again, by a suitable parameter refitting one might be able to describe the total cross sections, but given the more sophisticated scheme developed here, such approximations are no longer necessary.

\section{Outlook}

The present analysis of kaon photoproduction only made use of the minimal form of an amplitude subject to the constraints of two-body (coupled channel) unitarity and gauge-invariance. As expected, we could not produce a perfect fit to all the available experimental data, but nonetheless the result was encouraging, and it seems worthwile to work on improvements of the minimal ansatz for the amplitude. The most important building block is the amplitude for meson-baryon scattering, given by the solution of a BSE with a given kernel. So far, this kernel was given only by the Weinberg-Tomozawa term, and it is not surprising that our description of meson-baryon scattering is not yet satisfactory in most channels. It is straightforward (though still requiring quite some amount of work) to implement higher-order contact terms from the next-to-leading order mesonbaryon Lagrangian in the kernel (of course, this will also lead to additional photon-couplings by the minimal substitution rule). In principle, also the leading order Born graphs will have to be included in the meson-baryon potential. Work in this direction is currently under way.

Having achieved a suitable extension of the meson-baryon scattering amplitude, the photoproduction amplitude can be worked out along the lines displayed pictorially in figs. 2 and 3. Furthermore, the coupling of the photon to the mesons and baryons will have to be supplemented with higherorder terms, like e.g. couplings proportional to $\sigma^{\mu v} F_{\mu v}$. We are confident that refined versions of the model amplitude presented in this contribution will produce sensible results in the near- 


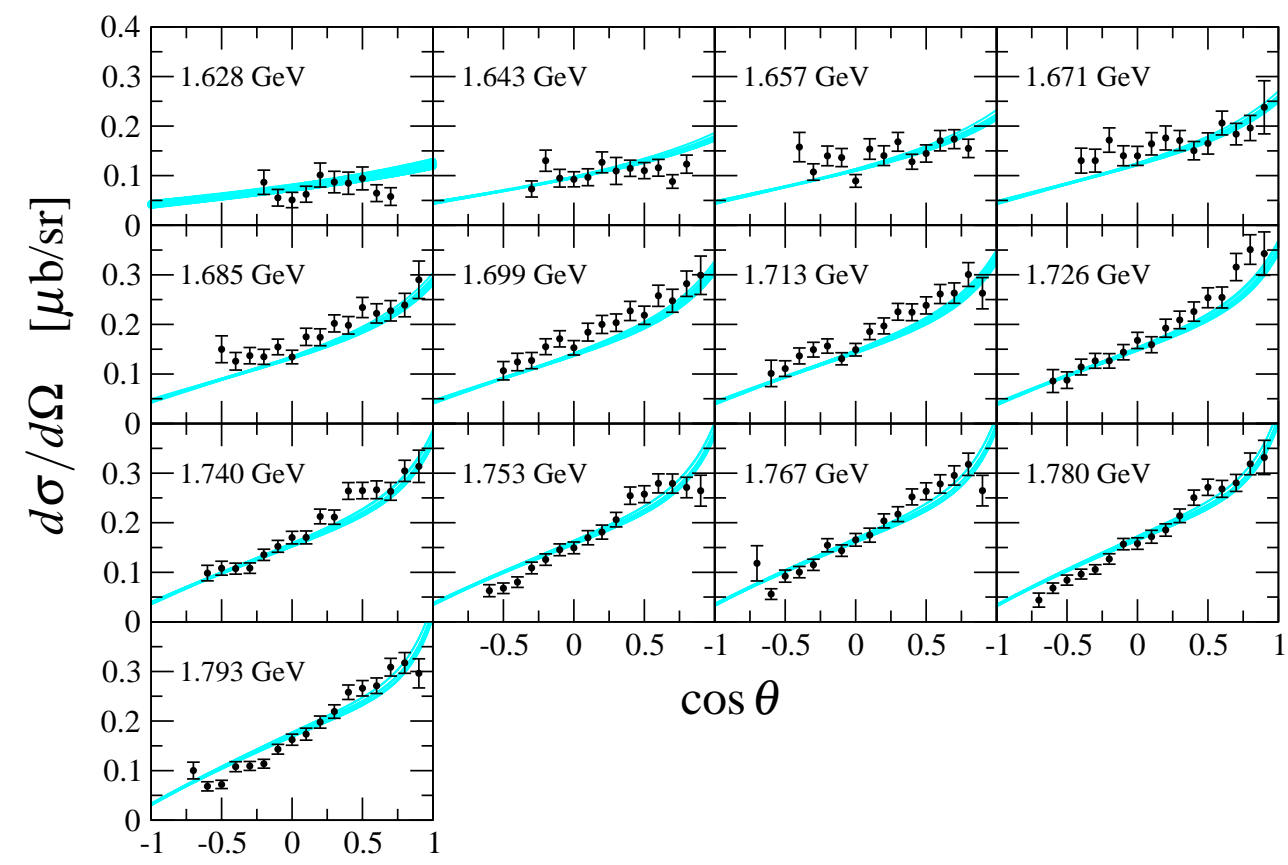

Figure 5: Differential cross sections for $\gamma p \rightarrow K^{+} \Lambda$ compared to data from [17]. The number in each plot denotes the respective c.m. energy $\sqrt{s}$.

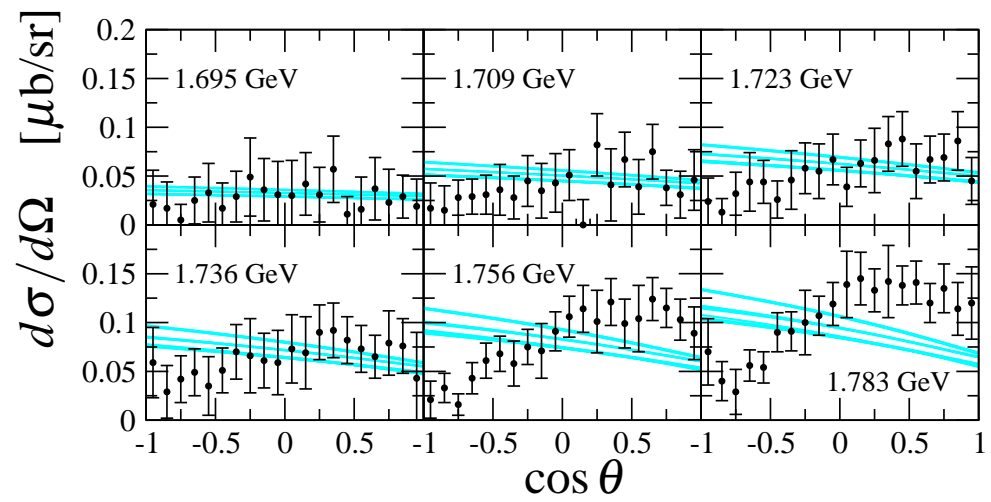

Figure 6: Differential cross sections for $\gamma p \rightarrow K^{+} \Sigma^{0}$ compared to data from [18]. The number in each plot denotes the respective c.m. energy $\sqrt{s}$. 
threshold regions, and will yield an important contribution to the theoretical understanding of the very complicated process of low-energy kaon photoproduction.

\section{References}

[1] N. Kaiser, P. B. Siegel and W. Weise, Phys. Lett. B 362 (1995) 23 [arXiv:nucl-th/9507036]; N. Kaiser, P. B. Siegel and W. Weise, Nucl. Phys. A 594 (1995) 325 [arXiv:nucl-th/9505043].

[2] J. A. Oller and U.-G. Meißner, Phys. Lett. B 500 (2001) 263 [arXiv:hep-ph/0011146]; U.-G. Meißner and J. A. Oller, Nucl. Phys. A 673 (2000) 311 [arXiv:nucl-th/9912026].

[3] J. A. Oller, E. Oset and A. Ramos, Prog. Part. Nucl. Phys. 45 (2000) 157 [arXiv:hep-ph/0002193].

[4] E. Oset and A. Ramos, Nucl. Phys. A 635 (1998) 99 [arXiv:nucl-th/9711022].

[5] M. F. M. Lutz and E. E. Kolomeitsev, Nucl. Phys. A 700 (2002) 193 [arXiv:nucl-th/0105042].

[6] N. Kaiser, T. Waas and W. Weise, Nucl. Phys. A 612 (1997) 297 [arXiv:hep-ph/9607459]; J. Caro Ramon, N. Kaiser, S. Wetzel and W. Weise, Nucl. Phys. A 672 (2000) 249 [arXiv:nucl-th/9912053].

[7] S. D. Bass, S. Wetzel and W. Weise, Nucl. Phys. A 686 (2001) 429 [arXiv:hep-ph/0007293].

[8] B. Borasoy, E. Marco and S. Wetzel, Phys. Rev. C 66 (2002) 055208 [arXiv:hep-ph/0212256].

[9] J. C. Nacher, E. Oset, H. Toki and A. Ramos, Phys. Lett. B 455 (1999) 55 [arXiv:nucl-th/9812055].

[10] J. C. Nacher, E. Oset, H. Toki and A. Ramos, Phys. Lett. B 461 (1999) 299 [arXiv:nucl-th/9902071].

[11] J. Caro Ramon, N. Kaiser, S. Wetzel and W. Weise, Nucl. Phys. A 672 (2000) 249 [arXiv:nucl-th/9912053].

[12] B. Borasoy, P. C. Bruns, U.-G. Meißner and R. Nißler, Eur. Phys. J. A 34 (2007) 161 [arXiv:0709.3181 [nucl-th]].

[13] F. E. Close and R. G. Roberts, Phys. Lett. B 316 (1993) 165 [arXiv:hep-ph/9306289]; B. Borasoy, Phys. Rev. D 59 (1999) 054021 [arXiv:hep-ph/9811411].

[14] P. C. Bruns, Ph.D. Thesis, Univ. Bonn, 2009 (http://hss.ulb.uni-bonn.de/diss_online).

[15] B. Borasoy, P. C. Bruns, U.-G. Meißner and R. Nißler, Phys. Rev. C 72 (2005) 065201 [arXiv:hep-ph/0508307].

[16] A. V. Anisovich, V. Kleber, E. Klempt, V. A. Nikonov, A. V. Sarantsev and U. Thoma, arXiv:0707.3596 [hep-ph].

[17] R. Bradford et al. [CLAS Collaboration], Phys. Rev. C 73 (2006) 035202 [arXiv:nucl-ex/0509033].

[18] K. H. Glander et al., Eur. Phys. J. A 19 (2004) 251 [arXiv:nucl-ex/0308025]. 